Journal of Mathematics and Informatics

Vol. 10, 2017, 21-29

ISSN: 2349-0632 (P), 2349-0640 (online)

Published 11 December 2017

www.researchmathsci.org

DOI: http://dx.doi.org/10.22457/jmi.v10a3

Journal of

Mathematics and

Informatics

\title{
On Triples where the Sum of any Two Members of a Triple is a Perfect Square
}

\author{
S.Vidhyalakshmi ${ }^{1}$,M.A.Gopalan ${ }^{2}$ and S. Aarthy Thangam ${ }^{3}$ \\ ${ }^{1,2}$ Department of Mathematics, Shrimati Indira Gandhi College \\ Trichy-620 002, Tamil Nadu, India. \\ ${ }^{3}$ Department of Mathematics, Shrimati Indira Gandhi College \\ Trichy-620 002, Tamil Nadu, India.
}

Received 15 November 2017; accepted 4 December 2017

Abstract. This paper deals with the construction of families of integer triples where, in each triple, the sum of any two members is a perfect square. A few numerical examples are also given.

Keywords: Integer pairs, integer triples

AMS Mathematics Subject Classification (2010): 11D99, 11D09

\section{Introduction}

Every advanced under-graduate and graduate student of mathematics as well as any researcher in number theory is familiar with Pythagorean triple which provides the relation between three sides of a right-angled triangle in addition to the concept of three integers representing an arithmetic progression, geometric progression and harmonic progression respectively. In this context, one may refer ${ }^{[1]}$ wherein the authors have given a collection of problems with solutions on integer triples in arithmetic progression.

Similar to a Pythagorean triple, we have a triple known as Heronian triple defined as follows: If $a, b, c$ represent the sides of a triangle with integer area, then the triple $(\mathrm{a}, \mathrm{b}, \mathrm{c})$ is known as Heronian triple. It is worth to note that not every Heronian triple is a Pythagorean triple. For example: $(4,13,15)$ is a Heronian triple but not Pythagorean triple whereas $(5,12,13)$ is both Heronian triple as well as Pythagorean triple. Also, we have a triple known as Eisenstein triple which is a set of integers which are the lengths of the sides of a triangle where one of the angle is $60^{\circ}$. In other words, An Eisenstein triple $(\mathrm{a}, \mathrm{b}, \mathrm{c})$ consists of three positive integers $a<c<b$ such that $a^{2}-a b+b^{2}=c^{2}$

No doubt that the triples in integers may be formulated in varieties of ways. For a review of various problems on triples, one may refer ${ }^{[2-6]}$. It is therefore towards this end, we are motivated to search for families of triples where, in each triple, the sum of any two of its members is a perfect square.

\section{Construction of triples}

Consider the Pythagorean equation given by 
S. Vidhyalakshmi, M.A. Gopalan and S. Aarthy Thangam

$$
x^{2}+y^{2}=z^{2}
$$

Observe that (1.1) is satisfied by

$$
x=m^{2}-n^{2}, \quad y=2 m n, \quad z=m^{2}+n^{2}, \quad m>n>0
$$

We present below different families of triples satisfying the required conditions.

\subsection{TRIPLE 1}

Assume

$$
\begin{aligned}
& x=6 p-3 \\
& y=6 q+2
\end{aligned}
$$

From (1.2), (1.3) and (1.4), we have

$$
p=\frac{1}{6}\left[m^{2}-n^{2}+3\right], \quad q=\frac{1}{3}[m n-1]
$$

The values of $\mathrm{p}$ and $\mathrm{q}$ are integers for the following choices:

$$
\begin{array}{ll}
\text { i) } & m=6 r-2, \quad n=6 s-5 \\
\text { ii) } & m=6 r-1, \quad n=6 s-4 \\
\text { iii) } & m=6 r+1, \quad n=6 s-2 \\
\text { iv) } & m=6 r+2, \quad n=6 s-1, \quad r \geq s \geq 1
\end{array}
$$

Choice : (i) $(m=6 r-2, \quad n=6 s-5)$

Substituting the values $m=6 r-2, n=6 s-5$ in (1.5), we have

$$
\begin{aligned}
& p=6 r^{2}-6 s^{2}-4 r+10 s-3=f_{1}(r, s) \\
& q=12 r s-10 r-4 s+3=g_{1}(r, s)
\end{aligned}
$$

Let $a_{1}(r, s)=\left(6 f_{1}(r, s)-3\right)^{2}, \quad b_{1}(r, s)=\left(6 g_{1}(r, s)+2\right)^{2}$

$\Rightarrow a_{1}(r, s)+b_{1}(r, s)$ is a perfect square.

Let $c_{1}(r, s)$ be any non-zero integer distinct from $a_{1}(r, s), b_{1}(r, s)$ such that

$$
\begin{aligned}
& a_{1}(r, s)+c_{1}(r, s)=\alpha^{2} \\
& b_{1}(r, s)+c_{1}(r, s)=\beta^{2}
\end{aligned}
$$

Subtraction of (1.7) from (1.6) gives

Employing the identity

$$
\alpha^{2}-\beta^{2}=a_{1}(r, s)-b_{1}(r, s)
$$

$$
(A+1)^{2}-A^{2}=2 A+1
$$

we have, from (1.8)

$$
A=\frac{1}{2}\left[a_{1}(r, s)-b_{1}(r, s)-1\right]
$$

where $\alpha=A+1, \quad \beta=A$

From (1.9) and (1.7), we have

$$
c_{1}(r, s)=\frac{1}{4}\left(\left[a_{1}(r, s)-b_{1}(r, s)-1\right]^{2}-4 b_{1}(r, s)\right)
$$

which is an integer for suitable values of $r$ and $s$. 
On triples where the sum of any two members of a triple is a perfect square

Hence, for those choices $\left(a_{1}(r, s), b_{1}(r, s), c_{1}(r, s)\right)$ is the required triple where the sum of any two of them is a perfect square.

Table 1: Numerical examples

\begin{tabular}{|c|c|c|c|c|c|c|r|}
\hline$r$ & $s$ & $a_{1}$ & $b_{1}$ & $c_{1}$ & $a_{1}+c_{1}$ & $b_{1}+c_{1}$ & $a_{1}+b_{1}$ \\
\hline 2 & 1 & 9801 & 400 & 22089600 & $4701^{2}$ & $4700^{2}$ & $101^{2}$ \\
\hline 3 & 2 & 42849 & 50176 & 13374720 & $3663^{2}$ & $3664^{2}$ & $305^{2}$ \\
\hline 5 & 3 & 378225 & 529984 & 5757244416 & $75879^{2}$ & $75880^{2}$ & $953^{2}$ \\
\hline 4 & 2 & 189225 & 94864 & 2225857536 & $47181^{2}$ & $47180^{2}$ & $533^{2}$ \\
\hline 3 & 3 & 7569 & 173056 & 6846396480 & $82743^{2}$ & $82744^{2}$ & $425^{2}$ \\
\hline
\end{tabular}

In a similar manner, the triples for choices (ii), (iii), (iv) are respectively given below in Tables 2, 3, 4 .

Choice : (ii) $m=6 r-1, \quad n=6 s-4$

Table 2: Numerical examples

\begin{tabular}{|c|c|c|c|c|c|c|r|}
\hline$r$ & $s$ & $a_{1}$ & $b_{1}$ & $c_{1}$ & $a_{1}+c_{1}$ & $b_{1}+c_{1}$ & $a_{1}+b_{1}$ \\
\hline 3 & 2 & 50625 & 73984 & 136348416 & $11679^{2}$ & $11680^{2}$ & $353^{2}$ \\
\hline 5 & 3 & 416025 & 659344 & 14800496256 & $121659^{2}$ & $121660^{2}$ & $1037^{2}$ \\
\hline 4 & 1 & 275625 & 8464 & 17843607936 & $133581^{2}$ & $133580^{2}$ & $533^{2}$ \\
\hline 4 & 2 & 216225 & 135424 & 1632024576 & $40401^{2}$ & $40400^{2}$ & $593^{2}$ \\
\hline 3 & 3 & 8649 & 226576 & 11872926720 & $108963^{2}$ & $108964^{2}$ & $485^{2}$ \\
\hline
\end{tabular}

Choice : (iii) $m=6 r+1, \quad n=6 s-2$

Table 3: Numerical examples

\begin{tabular}{|c|c|c|c|c|c|c|c|}
\hline$r$ & $s$ & $a_{1}$ & $b_{1}$ & $c_{1}$ & $a_{1}+c_{1}$ & $b_{1}+c_{1}$ & $a_{1}+b_{1}$ \\
\hline 2 & 1 & 23409 & 10816 & 39628800 & $6297^{2}$ & $6296^{2}$ & $185^{2}$ \\
\hline 3 & 2 & 68121 & 144400 & 1454515200 & $38139^{2}$ & $38140^{2}$ & $461^{2}$ \\
\hline 5 & 3 & 497025 & 984064 & 59301006336 & $243519^{2}$ & $243520^{2}$ & $1217^{2}$ \\
\hline 4 & 2 & 275625 & 250000 & 163897344 & $12813^{2}$ & $12812^{2}$ & $725^{2}$ \\
\hline 3 & 3 & 11025 & 369664 & 32155292736 & $179319^{2}$ & $179320^{2}$ & $617^{2}$ \\
\hline
\end{tabular}

Choice : (iv) $m=6 r+2, \quad n=6 s-1$

Table 4: Numerical examples

\begin{tabular}{|c|c|c|c|c|c|c|c|}
\hline$r$ & $s$ & $a_{1}$ & $b_{1}$ & $c_{1}$ & $a_{1}+c_{1}$ & $b_{1}+c_{1}$ & $a_{1}+b_{1}$ \\
\hline 2 & 1 & 29241 & 19600 & 23212800 & $4821^{2}$ & $4820^{2}$ & $221^{2}$ \\
\hline 3 & 2 & 77841 & 193600 & 3349900800 & $57879^{2}$ & $57880^{2}$ & $521^{2}$ \\
\hline 5 & 3 & 540225 & 1183744 & 103528313856 & $321759^{2}$ & $321760^{2}$ & $1313^{2}$ \\
\hline 4 & 2 & 308025 & 327184 & 91449216 & $9579^{2}$ & $9580^{2}$ & $797^{2}$ \\
\hline 3 & 3 & 12321 & 462400 & 50642539200 & $225039^{2}$ & $225040^{2}$ & $689^{2}$ \\
\hline
\end{tabular}


S. Vidhyalakshmi, M.A. Gopalan and S. Aarthy Thangam

\subsection{TRIPLE 2}

Assume

$$
\begin{aligned}
& x=6 p-3 \\
& y=4 q+4
\end{aligned}
$$

From (1.2), (1.10) and (1.11), we have

$$
p=\frac{1}{6}\left[m^{2}-n^{2}+3\right], \quad q=\frac{1}{2}[m n-2]
$$

The values of $\mathrm{p}$ and $\mathrm{q}$ are integers when

$$
m=6 r+s-3, \quad n=s
$$

where $r, s \in Z-\{0\}$

Substituting (1.13) in (1.12), we have

$$
\begin{aligned}
& p=6 r^{2}-6 r+2 r s-s+2=f_{2}(r, s) \\
& q=\frac{1}{2}\left(s^{2}+6 r s-3 s-2\right)=g_{2}(r, s)
\end{aligned}
$$

Let $a_{2}(r, s)=\left(6 f_{2}(r, s)-3\right)^{2}, \quad b_{2}(r, s)=\left(4 g_{2}(r, s)+4\right)^{2}$

$$
\Rightarrow a_{2}(r, s)+b_{2}(r, s) \text { is a perfect square. }
$$

Let $c_{2}(r, s)$ be any non-zero integer distinct from $a_{2}(r, s), b_{2}(r, s)$ such that

$$
\begin{aligned}
& a_{2}(r, s)+c_{2}(r, s)=\alpha^{2} \\
& b_{2}(r, s)+c_{2}(r, s)=\beta^{2}
\end{aligned}
$$

Following the procedure as in triple: 1 , it is seen that

$$
c_{2}(r, s)=\frac{1}{4}\left(\left[a_{2}(r, s)-b_{2}(r, s)-1\right]^{2}-4 b_{2}(r, s)\right)
$$

which is an integer for suitable values of $\mathrm{r}$ and $\mathrm{s}$.

Hence, for those choices $\left(a_{2}(r, s), b_{2}(r, s), c_{2}(r, s)\right)$ is the required triple such that the sum of any two of them is a perfect square.

Table 5: Numerical Examples

\begin{tabular}{|c|c|c|c|c|c|c|c|}
\hline$r$ & $s$ & $a_{2}$ & $b_{2}$ & $c_{2}$ & $a_{2}+c_{2}$ & $b_{2}+c_{2}$ & $a_{2}+b_{2}$ \\
\hline 2 & 3 & 18225 & 5184 & 42505216 & $6521^{2}$ & $6520^{2}$ & $153^{2}$ \\
\hline 3 & 5 & 140625 & 40000 & 2531257344 & $50313^{2}$ & $50312^{2}$ & $425^{2}$ \\
\hline 4 & 2 & 275625 & 8464 & 17843607936 & $133581^{2}$ & $133580^{2}$ & $533^{2}$ \\
\hline 5 & 2 & 700569 & 13456 & 118030711680 & $343557^{2}$ & $343556^{2}$ & $845^{2}$ \\
\hline 2 & 2 & 13689 & 1936 & 34525440 & $5877^{2}$ & $5876^{2}$ & $125^{2}$ \\
\hline
\end{tabular}

\subsection{TRIPLE 3}

Assume

$$
\begin{aligned}
& x=3 p-3 \\
& y=6 q+2
\end{aligned}
$$

From (1.2), (1.14) and (1.15), we have 
On triples where the sum of any two members of a triple is a perfect square

$$
p=\frac{1}{3}\left[m^{2}-n^{2}+3\right], \quad q=\frac{1}{3}[m n-1]
$$

The values of $\mathrm{p}$ and $\mathrm{q}$ are integers for the following choices:

$$
\begin{array}{ll}
\text { i) } & m=3 r+3 s-2, \quad n=3 r-2 \\
\text { ii) } & m=3 r+3 s-1, \quad n=3 r-1
\end{array}
$$

where $r, s \in Z-\{0\}$.

Choice : (i) $(m=3 r+3 s-2, \quad n=3 r-2)$

Substituting the values $m=3 r+3 s-2, \quad n=3 r-2$ in (1.16), we have

$$
\begin{aligned}
& p=3 s^{2}+6 r s-4 s+1=f_{3}(r, s) \\
& q=3 r^{2}-4 r-2 s+3 r s+1=g_{3}(r, s)
\end{aligned}
$$

Let $a_{3}(r, s)=9\left(f_{3}(r, s)-1\right)^{2}, \quad b_{3}(r, s)=4\left(3 g_{3}(r, s)+1\right)^{2}$

$$
\Rightarrow a_{3}(r, s)+b_{3}(r, s) \text { is a perfect square. }
$$

Let $c_{3}(r, s)$ be any non-zero integer distinct from $a_{3}(r, s), b_{3}(r, s)$ such that

$$
\begin{aligned}
& a_{3}(r, s)+c_{3}(r, s)=\alpha^{2} \\
& b_{3}(r, s)+c_{3}(r, s)=\beta^{2}
\end{aligned}
$$

After performing a few calculations, it is seen that

$$
c_{3}(r, s)=\frac{1}{4}\left(\left[a_{3}(r, s)-b_{3}(r, s)-1\right]^{2}-4 b_{3}(r, s)\right)
$$

which is an integer for suitable values of $r$ and $s$.

Hence for those choices $\left(a_{3}(r, s), b_{3}(r, s), c_{3}(r, s)\right)$ is the required triple such that the sum of any two of them is a perfect square.

Table 6: Numerical examples

\begin{tabular}{|c|c|c|c|c|c|c|c|}
\hline$r$ & $s$ & $a_{3}$ & $b_{3}$ & $c_{3}$ & $b_{3}+c_{3}$ & $a_{3}+c_{3}$ & $a_{3}+b_{3}$ \\
\hline 2 & 1 & 1089 & 3136 & 1045440 & $1024^{2}$ & $1023^{2}$ & $652^{2}$ \\
\hline 5 & 3 & 99225 & 327184 & 12991113216 & $113980^{2}$ & $113979^{2}$ & $653^{2}$ \\
\hline 3 & 3 & 42849 & 50176 & 13374720 & $3664^{2}$ & $3663^{2}$ & $305^{2}$ \\
\hline 3 & 1 & 2601 & 19600 & 72230400 & $8500^{2}$ & $8499^{2}$ & $149^{2}$ \\
\hline
\end{tabular}

In a similar manner, the triple for choice (ii) is given in Table: 7

Choice : (ii) $m=3 r+3 s-1, \quad n=3 r-1$. 
S. Vidhyalakshmi, M.A. Gopalan and S. Aarthy Thangam

Table 7: Numerical examples

\begin{tabular}{|c|c|c|c|c|c|c|c|}
\hline$r$ & $s$ & $a_{3}$ & $b_{3}$ & $c_{3}$ & $a_{3}+c_{3}$ & $b_{3}+c_{3}$ & $a_{3}+b_{3}$ \\
\hline 2 & 1 & 1521 & 6400 & 5947200 & $2439^{2}$ & $2440^{2}$ & $89^{2}$ \\
\hline 5 & 3 & 110889 & 414736 & 23080487040 & $151923^{2}$ & $151924^{2}$ & $725^{2}$ \\
\hline 3 & 3 & 50625 & 73984 & 136348416 & $11679^{2}$ & $11680^{2}$ & $353^{2}$ \\
\hline 3 & 1 & 3249 & 30976 & 192179520 & $13863^{2}$ & $13864^{2}$ & $185^{2}$ \\
\hline
\end{tabular}

\subsection{TRIPLE 4}

Assume

$$
\begin{aligned}
& x=5 p-5 \\
& y=2 q+2
\end{aligned}
$$

From (1.2), (1.17) and (1.18), we have

$$
p=\frac{1}{5}\left[m^{2}-n^{2}+5\right], \quad q=m n-1
$$

The values of $\mathrm{p}$ and $\mathrm{q}$ are integers for the following choices:
i) $m=2 r+5 s-1, \quad n=2 r-1$
ii) $m=3 r+5 s-4, \quad n=2 r-1$
iii) $m=3 r+5 s-5, \quad n=2 r$
iv) $m=2 r+5 s, \quad n=2 r, r \geq s \geq 1$

Choice : (i) $m=2 r+5 s-1, \quad n=2 r-1$

Substituting the values $m=2 r+5 s-1, \quad n=2 r-1$ in (1.19), we have

$$
\begin{aligned}
& p=5 s^{2}+4 r s-2 s+1=f_{4}(r, s) \\
& q=4 r^{2}-4 r-5 s+10 r s=g_{4}(r, s)
\end{aligned}
$$

Let $a_{4}(r, s)=\left(5 f_{4}(r, s)-5\right)^{2}, \quad b_{4}(r, s)=\left(2 g_{4}(r, s)+2\right)^{2}$

$\Rightarrow a_{4}(r, s)+b_{4}(r, s)$ is a perfect square.

Let $c_{4}(r, s)$ be any non-zero integer distinct from $a_{4}(r, s), b_{4}(r, s)$ such that

$$
\begin{aligned}
& a_{4}(r, s)+c_{4}(r, s)=\alpha^{2} \\
& b_{4}(r, s)+c_{4}(r, s)=\beta^{2}
\end{aligned}
$$

Subtraction of (1.21) from (1.20) gives

$$
\alpha^{2}-\beta^{2}=a_{4}(r, s)-b_{4}(r, s)
$$

Employing the identity

$$
(A+1)^{2}-A^{2}=2 A+1
$$

we have, from (1.22) 
On triples where the sum of any two members of a triple is a perfect square

$$
A=\frac{1}{2}\left[a_{4}(r, s)-b_{4}(r, s)-1\right]
$$

where $\alpha=A+1, \quad \beta=A$

From (1.23) and (1.21), we have

$$
c_{4}(r, s)=\frac{1}{4}\left(\left[a_{4}(r, s)-b_{4}(r, s)-1\right]^{2}-4 b_{4}(r, s)\right)
$$

which is an integer for suitable values of $\mathrm{r}$ and $\mathrm{s}$.

Hence, for those choices $\left(a_{4}(r, s), b_{4}(r, s), c_{4}(r, s)\right)$ is the required triple where the sum of any two of them is a perfect square.

Table 8: Numerical examples

\begin{tabular}{|c|c|c|c|c|c|c|c|}
\hline$r$ & $s$ & $a_{4}$ & $b_{4}$ & $c_{4}$ & $a_{4}+c_{4}$ & $b_{4}+c_{4}$ & $a_{4}+b_{4}$ \\
\hline 2 & 1 & 3025 & 2304 & 127296 & $361^{2}$ & $360^{2}$ & $73^{2}$ \\
\hline 5 & 3 & 245025 & 186624 & 852453376 & $29201^{2}$ & $29200^{2}$ & $657^{2}$ \\
\hline 3 & 3 & 140625 & 40000 & 2531257344 & $50313^{2}$ & $50312^{2}$ & $425^{2}$ \\
\hline 3 & 1 & 5625 & 10000 & 4777344 & $2187^{2}$ & $2188^{2}$ & $125^{2}$ \\
\hline
\end{tabular}

In a similar manner, the triples for choices (ii), (iii), (iv) are respectively given below in Tables 9, 10, 11.

Choice : (ii) $m=3 r+5 s-4, \quad n=2 r-1$

Table 9: Numerical examples

\begin{tabular}{|c|c|c|c|c|c|c|c|}
\hline$r$ & $s$ & $a_{4}$ & $b_{4}$ & $c_{4}$ & $a_{4}+c_{4}$ & $b_{4}+c_{4}$ & $a_{4}+b_{4}$ \\
\hline 5 & 3 & 354025 & 219024 & 4556030976 & $67501^{2}$ & $67500^{2}$ & $757^{2}$ \\
\hline 4 & 2 & 75625 & 63504 & 36660096 & $6061^{2}$ & $6060^{2}$ & $373^{2}$ \\
\hline 3 & 3 & 140625 & 40000 & 2531257344 & $50313^{2}$ & $50312^{2}$ & $425^{2}$ \\
\hline 4 & 4 & 540225 & 153664 & 37357004736 & $193281^{2}$ & $193280^{2}$ & $833^{2}$ \\
\hline
\end{tabular}

Choice : (iii) $m=3 r+5 s-5, \quad n=2 r$

Table 10: Numerical examples

\begin{tabular}{|c|c|c|c|c|c|c|c|}
\hline$r$ & $s$ & $a_{4}$ & $b_{4}$ & $c_{4}$ & $a_{4}+c_{4}$ & $b_{4}+c_{4}$ & $a_{4}+b_{4}$ \\
\hline 5 & 3 & 275625 & 250000 & 163897344 & $12813^{2}$ & $12812^{2}$ & $725^{2}$ \\
\hline 4 & 2 & 50625 & 73984 & 136348416 & $11679^{2}$ & $11680^{2}$ & $353^{2}$ \\
\hline 3 & 3 & 105625 & 51984 & 719260416 & $26821^{2}$ & $26820^{2}$ & $397^{2}$ \\
\hline 4 & 4 & 442225 & 186624 & 16332653376 & $127801^{2}$ & $127800^{2}$ & $793^{2}$ \\
\hline 3 & 1 & 2025 & 11664 & 23220736 & $4819^{2}$ & $4820^{2}$ & $117^{2}$ \\
\hline
\end{tabular}

Choice : (iv) $m=2 r+5 s, \quad n=2 r$ 
S. Vidhyalakshmi, M.A. Gopalan and S. Aarthy Thangam

Table 11: Numerical examples

\begin{tabular}{|c|c|c|c|c|c|c|c|}
\hline$r$ & $s$ & $a_{4}$ & $b_{4}$ & $c_{4}$ & $a_{4}+c_{4}$ & $b_{4}+c_{4}$ & $a_{4}+b_{4}$ \\
\hline 2 & 1 & 4225 & 5184 & 225216 & $479^{2}$ & $480^{2}$ & $97^{2}$ \\
\hline 5 & 3 & 275625 & 250000 & 163897344 & $12813^{2}$ & $12812^{2}$ & $725^{2}$ \\
\hline 3 & 3 & 164025 & 63504 & 2526004096 & $50261^{2}$ & $50260^{2}$ & $477^{2}$ \\
\hline 3 & 1 & 7225 & 17424 & 25992576 & $5099^{2}$ & $5100^{2}$ & $157^{2}$ \\
\hline
\end{tabular}

\subsection{TRIPLE 5}

Assume

$$
\begin{aligned}
& x=7 p-7 \\
& y=2 q+2
\end{aligned}
$$

From (1.2), (1.24) and (1.25), we have

$$
p=\frac{1}{7}\left[m^{2}-n^{2}+7\right], \quad q=m n-1
$$

The values of $\mathrm{p}$ and $\mathrm{q}$ are integers when

$$
m=r+7 s-7, \quad n=r \quad, r \geq s \geq 1
$$

Substituting (1.27) in (1.26), we have

$$
p=1+(s-1)(2 r+7 s-7)=f_{5}(r, s), q=r(r+7 s-7)-1=g_{5}(r, s)
$$

Let $a_{5}(r, s)=\left(7 f_{5}(r, s)-7\right)^{2}, \quad b_{5}(r, s)=\left(2 g_{5}(r, s)+2\right)^{2}$

$$
\Rightarrow a_{5}(r, s)+b_{5}(r, s) \text { is a perfect square. }
$$

Let $c_{5}(r, s)$ be any non-zero integer distinct from $a_{5}(r, s), b_{5}(r, s)$ such that

$$
\begin{aligned}
& a_{5}(r, s)+c_{5}(r, s)=\alpha^{2} \\
& b_{5}(r, s)+c_{5}(r, s)=\beta^{2}
\end{aligned}
$$

Subtraction of (1.29) from (1.28) gives

Employing the identity

$$
\alpha^{2}-\beta^{2}=a_{5}(r, s)-b_{5}(r, s)
$$

$$
(A+1)^{2}-A^{2}=2 A+1
$$

we have, from (1.30)

$$
A=\frac{1}{2}\left[a_{5}(r, s)-b_{5}(r, s)-1\right]
$$

where $\alpha=A+1, \quad \beta=A$

From (1.29) and (1.31), we have

$$
c_{5}(r, s)=\frac{1}{4}\left(\left[a_{5}(r, s)-b_{5}(r, s)-1\right]^{2}-4 b_{5}(r, s)\right)
$$

which is an integer for suitable values of $r$ and $s$. 
On triples where the sum of any two members of a triple is a perfect square

Hence, for those choices $\left(a_{5}(r, s), b_{5}(r, s), c_{5}(r, s)\right)$ is the required triple where the sum of any two of them is a perfect square.

Table 12: Numerical examples

\begin{tabular}{|c|c|c|c|c|c|c|c|}
\hline$r$ & $s$ & $a_{5}$ & $b_{5}$ & $c_{5}$ & $a_{5}+c_{5}$ & $b_{5}+c_{5}$ & $a_{5}+b_{5}$ \\
\hline 1 & 2 & 3969 & 256 & 3444480 & $1857^{2}$ & $1856^{2}$ & $65^{2}$ \\
\hline 5 & 4 & 423801 & 67600 & 31719542400 & $178101^{2}$ & $178100^{2}$ & $701^{2}$ \\
\hline 3 & 2 & 8281 & 3600 & 5472000 & $2341^{2}$ & $2340^{2}$ & $109^{2}$ \\
\hline 7 & 4 & 540225 & 153664 & 37357004736 & $193281^{2}$ & $193280^{2}$ & $833^{2}$ \\
\hline
\end{tabular}

\section{Conclusion}

In this paper, we have made an attempt to construct family of triples where the sum of any members of a triple is a perfect square. To conclude, one may search for families of triples with different relations among its members.

\section{REFERENCES}

1. M.A.Gopalan, K.Meena and S.Aarthy Thangam, Special Integer Triples In Arithmetic Progression With Solutions, Lambert Academic Publishing, OmniScriptum Publishing Group, Berlin, (2017).

2. V. Pandichelvi and P. Sivakamasundari, Construction of euler square involving some figurate numbers, International Journal of Multidisciplinary Research and Development, 3 (6) (2016) 40-43.

3. S.Vidhyalakshmi, M.A.Gopalan and E.Premalatha, Construction of two special integer triples, International Journal of Mathematics And its Applications, 4 (1-C) (2016) 103-106.

4. S.Vidhyalakshmi, M.A.Gopalan and E.Premalatha, An interesting diophantine problem on triples-V, Open Journal of Applied \& Theoretical Mathematics, 2 (2) (2016) 37-41.

5. M.A.Gopalan, S.Vidhyalakshmi, E.Premalatha and K.Agalya, An Interesting Diophantine problem on triples-III, International Journal of Emerging Technologies in Engineering Research, 3 (2) (2015) 47-49.

6. M.A.Gopalan, S.Vidhyalakshmi, V.Geetha and Rukmani, An interesting diophantine problem on triple-I, International Journal of Emerging Technologies in Engineering Research, 3 (2) (2015) 28-30. 